\title{
NEWS UPDATES
}

\section{Clock stops for hibernating hamsters}

When an animal hibernates, energy-costly processes such as heartbeat and respiration run far more slowly than usual. Paul Pévet and colleagues at Université Louis Pasteur (Strasbourg, France) investigated the brains of European hamsters to find out whether the same rule applies to the circadian clock.

European hamsters follow a typical hibernation pattern, consisting of periods of deep torpor, in which body temperature is low, followed by short periods of euthermia, or normal body temperature. The researchers sent hamsters into hibernation by transferring them to a cold room with a short, winter-like light/dark cycle (Proc. Natl. Acad. Sci. USA 104, 13816-13820; 2007). They then examined hamsters' circadian clock genes and gene output signals during the day and at night. In euthermic hamsters, clocks functioned the way they would in nonhibernating hamsters, fluctuating over the course of the day. Hamsters in deep torpor, however, showed no change in clock gene expression. This suggests that their circadian clocks ceased to function, though metabolic activity remained relatively high in the area of the brain where the master clock is located.

The team's findings are specific to hamsters, but may provide insight into human torpor-like states such as near-drowning in cold water and induced hypothermia for surgery.

\section{Brain morphology in capuchin monkeys}

New research shows that, like humans, capuchin monkeys may have certain differences in brain structure that are related to sex and 'handedness'. Kimberley Phillips (Hiram College, Hiram, $\mathrm{OH}$ ) and colleagues measured the size of the corpus callosum, the main white matter structure connecting the two cerebral hemispheres, in 14 capuchin monkeys. Nine of the monkeys were also given a test used to determine handedness (PLoS One 2, e792; 2007).

The corpus callosum was relatively larger in adult females than in males and was also relatively larger in left-handed monkeys than in 'righties'. There was also a significant interaction between sex and handedness, with right-handed males having a relatively smaller corpus callosum than right-handed females. These results are similar to the morphological sexbased and handedness-based differences observed in humans.

Sex differences in corpus callosum morphology have not been previously reported in any nonhuman primates studied so far. This research thus suggests for the first time that the influence of sex on brain structure may not be unique to humans. Because differences in spatial-analysis ability may be associated with sex differences in corpus callosum morphology in humans, more information is needed about spatial abilities of nonhuman primates to elucidate the functional importance of sex differences in corpus callosum morphology.

\section{Knockout mice don't need to diet}

Diet fads of recent years have attempted to capitalize on the apparent slimming effects of increasing protein intake. Though the mechanisms of these effects are still poorly understood, they are thought to be mediated at least in part by branched-chain amino acids such as leucine. Christopher Lynch and colleagues at the Pennsylvania State University College of Medicine (Hershey, PA) studied the effects of chronically elevated leucine concentrations by engineering mice to stop producing BCAT2, an enzyme that breaks down branched-chain amino acids.

Though modified mice consumed more food than wildtype mice and were equally active, they burned more calories and were leaner (Cell Metab. 6, 181-194; 2007). The extra energy expenditure seemed to result from a futile protein turnover cycle, in which unnecessary proteins were synthesized and then destroyed. Lean mice were also more glucose-tolerant and had higher sensitivity to insulin. When fed a high-fat diet, they did not become obese, whereas normal mice did.

Previous studies have not clearly shown whether an increased concentration of leucine is beneficial or detrimental in controlling obesity. The team's findings suggest that focusing on the metabolism of branched-chain amino acids, rather than the concentration, may be the key to treating obesity in humans. 